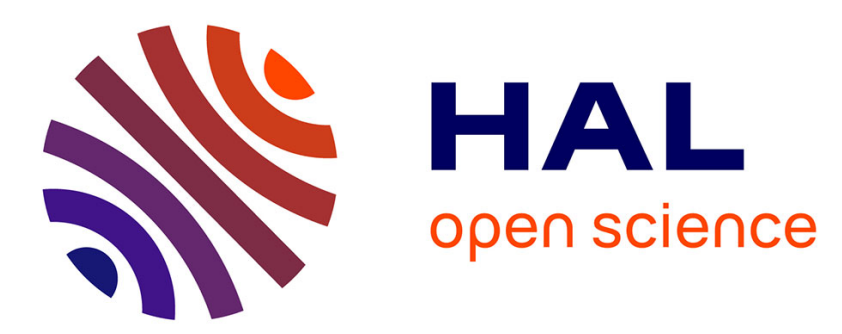

\title{
Effects of Sensitive Electrical Stimulation-Based Somatosensory Cueing in Parkinson's Disease Gait and Freezing of Gait Assessment
}

Benoît Sijobert, Christine Azevedo Coste, David Andreu, Claudia Verna, Christian Geny

\section{To cite this version:}

Benoît Sijobert, Christine Azevedo Coste, David Andreu, Claudia Verna, Christian Geny. Effects of Sensitive Electrical Stimulation-Based Somatosensory Cueing in Parkinson's Disease Gait and Freezing of Gait Assessment. Artificial Organs, 2017, 41 (11), pp.E222-E232. 10.1111/aor.13059 . hal01638837

\section{HAL Id: hal-01638837 https://hal.inria.fr/hal-01638837}

Submitted on 20 Nov 2017

HAL is a multi-disciplinary open access archive for the deposit and dissemination of scientific research documents, whether they are published or not. The documents may come from teaching and research institutions in France or abroad, or from public or private research centers.
L'archive ouverte pluridisciplinaire HAL, est destinée au dépôt et à la diffusion de documents scientifiques de niveau recherche, publiés ou non, émanant des établissements d'enseignement et de recherche français ou étrangers, des laboratoires publics ou privés. 


\section{Effects of sensitive electrical stimulation-based somatosensory cueing in Parkinson's disease gait and Freezing of Gait assessment}

Benoît SIJOBERT, Christine AZEVEDO, David ANDREU, Claudia VERNA, and Christian GENY

Abstract - Objectives. This study aims to investigate the effect of a somatosensory cueing on gait disorders in subjects with Parkinson's Disease (PD). Materials and Methods. After having performed stepping in place (SIP) and timed up and go (TUG) assessing tasks, 13 participants with PD were equipped with an electrical stimulator and an inertial measurement unit (IMU) located under the lateral malleolus on the sagittal plane. Electrodes were positioned under the arch of the foot and electrical stimulation (ES) parameters (five $500 \mu \mathrm{s} /$ phase charge-balanced biphasic pulses delivered at $200 \mathrm{~Hz}$, repeated 4 times at $10 \mathrm{~Hz}$ ) adjusted to deliver a sensitive signal. Online IMU signal was processed in order to trigger ES at heel off detection. Starting from a quiet standing posture, subjects were asked to walk at their preferred speed on a path including $5 \mathrm{~m}$ straight line, u-turn and walk around tasks. 3 situations were considered: no stimulation baseline pre-condition $(\mathrm{C} 0)$, ES condition $(\mathrm{C} 1)$, no stimulation baseline postcondition (CObis), for eliminating a learning effect possibility. Results. In ES condition (C1) the time to execute the different tasks was globally decreased in all the subjects $(n=13)$. Participants' results were then grouped regarding whether they experienced Freezing of Gait (FOG) or not during C0 no stimulation baseline pre-condition. In "freezer" subjects $(n=9)$, the time to complete the entire path was reduced by $19 \%$. FOG episodes occurrence was decreased by $12 \%$ compared to baseline conditions. Conclusion. This preliminary work showed a positive global effect on gait and FOG in PD of a somatosensory cueing based on sensitive electrical stimulation.

\section{INTRODUCTION}

Parkinson's disease (PD) is the second most common neurodegenerative disorder. It affects approximately ten million people worldwide, among them essentially adults over 60 years old [1][2]. The number of persons suffering from PD has been increasing with the aging population. Various symptoms having an important impact on quality of life are associated to PD, such as tremor, bradykinesia and gait impairment leading to an increased risk of falls [3]. Patients with Parkinson's disease can be subject to a particular paroxysmal gait impairment called freezing of gait (FOG) and defined by Heremans [4] as "a brief, episodic absence or marked reduction of forward progression of the feet despite the intention to walk". FOG can occur in different daily life situations and especially at gait initiation, turning back, standing up from a chair, walking over changes in floor patterns or going through narrow spaces [5][7]. This peculiar symptom could be disabling, leading to falls and remains poorly influenced by dopaminergic drugs. Previous studies have shown that visual or auditory stimuli can help individuals with PD to reduce the occurrence and duration of FOG events [8]-[10] thereby improving their gait [10][11]. In their meta-analysis, Spaulding et al. [13] reviewed the numerous studies on visual and auditory cueing by comparing their efficacy on gait. They concluded to a benefit of auditory cueing on velocity, stride length and cadence; while visual cueing only resulted in stride length changes. Nevertheless, auditory and visual cueing modalities appear to be effective only in experimental 
conditions and with bystander effect [14]. It has been also shown that variability in beat perception between subjects could differently affect gait when synchronizing footsteps to music or metronome cues [15].

The effects of sensorimotor cueing are not clearly established because this stimulation modality has received little attention comparatively to other ones. Numerous studies confirmed that Parkinson's disease motor deficits are associated with proprioceptive impairments. Vaugoyeau et al. [16] analyzed the postural adjustments of PD subjects standing on a platform executing small angular sinusoidal oscillations. Subjects were asked to maintain a vertical posture. In the absence of visual cues, subjects were clearly unable to use proprioceptive information as feedback to control their body verticality and stabilize their body segments, resulting in blocking head and shoulders segments. The same strategies have been observed during their gait [17]. The authors concluded that sensorimotor integration deficits partly account for the postural and locomotion impairments observed in PD. Other studies have shown that gait in PD patients could be improved by increasing somatosensory information from the plantar surface of the feet using textured insoles [18]. Rhythmic somatosensory cueing (RSC) has also been investigated [14]. Authors used a miniature-vibrating cylinder attached to the wrist as a cueing device in 17 patients. RSC improved the gait with lower stride frequencies and larger step lengths, while maintaining walking speed. This study demonstrated that patients with PD could dynamically modify their stepping pattern to adapt to an external stimulus using somatosensory pathways. RSC seems to have a robust effect in gait pattern and to be resistant to visual interferences. Authors suggested that this technique could be a viable alternative to auditory or visual cueing.

To our knowledge, only three studies involving cutaneous electrical stimulation (ES) applied on patients with PD have been published. Mann et al. [19] studied the feasibility of functional electrical stimulation (FES) to assist gait in PD. During eight weeks they performed walking sessions under electrical stimulation of the common peroneal nerve of the more affected leg in 6 subjects. Stimulation was triggered by a pressure-sensitive switch in the shoe and set to gain effective dorsiflexion and eversion of the foot during walking similarly to a drop foot stimulation modality. An immediate improvement was demonstrated with FES on distance and average stride length during a 3-min walk but not on the number of steps and walking speed. Fewer episodes of FOG occurred during the treatment period. Similarly, Popa et al.[20] used FES to assist dorsiflexion on 11 PD subjects during two weeks. They noticed a slight increase in step length and cadence. In the third study [21], the same stimulation approach was applied on nine PD patients. Results showed a decreased duration of double support phase and variability of stride duration and stride length with FES. Two subjects did not experience FOG in a few situations where they previously experienced some. Using muscle vibration during voluntary dorsiflexion movements of the ankle joint, Khudados et al. [22] showed that proprioceptive regulation of voluntary movement is disturbed in PD. El-Tamawy et al. [23] used augmented proprioceptive cues during gait on thirty levodopa-dependent PD subjects. They applied vibratory stimuli to the feet plantar surfaces (below the heel and forefoot) through miniature hidden vibrating devices that sent rhythmic 
vibrations to the skin in synchronization with the step at the push off-phase of the gait. Results demonstrated a significant improvement in gait kinematics and angular excursion of lower limb joints. Similarly, Kleiner et al. [24] applied mechanical stimulation (AMPS: Automated Mechanical Peripheral Stimulation Treatment) on four specific target areas in patient's feet while they were laying down and reported a $15 \%$ improvement in gait velocity after treatment.

From these previous statements, we decided to design a protocol based on assessing the effects of somatosensory cueing by sensitive electrical stimulation. Most of the published studies have assessed the effect of cueing only during straight walking, in experimental conditions and with severe dopamine deficiency. However, FOG occurs in a random manner, in different environments, depending from the dopaminergic and emotional status.

In both on and off conditions (under medication or not), turning phase has been demonstrated as the most frequent trigger of freezing of gait in Parkinson's disease. In their study, Schaafsma et al [25] found that FOG was mainly elicited by turns (63\%), first step (23\%), walking through narrow spaces (12\%) and reaching destinations $(9 \%)$. We also observed this predominance in some of our previous experiments [26]. Plotnik et al. tried to explain this occurrence by the asymmetric nature of these tasks, which would increase interlimb synchronization difficulties [27]. Crenna et al. showed this could possibly be related to a head rotation control. Indeed, patients in the early stage of the disease initiate head rotation later than controls while turning [28]. Nieuwboer et al. [29] chose to focus their work on functional turning performance with different cueing modalities and observed it improved the turning speed in all subjects.

In order to increase FOG occurrence during experimentations and to be close to the daily life situations, we designed an experimental path including a maximum of turning phases. We have also associated to our experimental protocol a postural task developed by Nantel et al. [30]: the repetitive stepping in place (SIP). Previously used in other studies to evoke turning in healthy subjects [31], the authors showed that the SIP task was correlated with the score of selfassessment questionnaire (FOG-Q) and could be an objective measurement of FOG. This feature led us to test this paradigm as a possible tool for assessing efficiency of a cueing method.

\section{MATERIAL AND METHOD}

\section{A. Stimulation}

Inspired by Spaich work on hemiparetic gait [32], we stimulated the arch of the foot as shown in Fig. 1. A selfadhesive electrode $\left(2.6 \mathrm{~cm}^{2}\right)$ was placed as the cathode on the arch of the foot and a large common anode $\left(45 \mathrm{~cm}^{2}\right)$ was placed on the dorsum of the foot. The stimulation pattern consisted in five $500 \mu$ s/phase charge-balanced biphasic pulses delivered at $200 \mathrm{~Hz}$, repeated 4 times at $10 \mathrm{~Hz}$. Current amplitude was adjusted in order for the subject to feel the stimulation without any discomfort. Our initial hypothesis was to trigger a withdrawal reflex for 
helping to elicit gait at heel off. However the needed stimulation levels for enabling the reflex were too high and even at high intensities no withdrawal was triggered in some patients. We decided thus to stay at a sensitive and not motor stimulation level. Subjects were equipped with one inertial measurement unit (HikoB ${ }^{\odot}$ Villeurbanne, France) strapped to the foot and a wirelessly programmable electro-stimulator (Phenix $\odot$ Neo Usb, Montpellier, France) strapped around the shank.

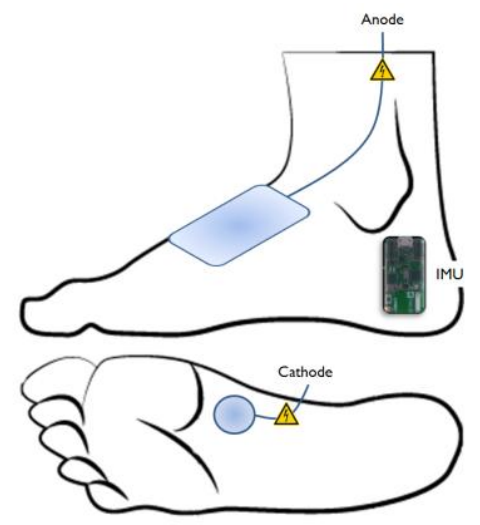

Figure 1 Electrodes and IMU locations. To deliver the stimulation, a self-adhesive electrode is placed as the cathode on the arch of the foot and a common anode is placed on the dorsum of the foot.

\section{B. Inertial Measurement Unit based triggering}

People with advanced Parkinson's disease are usually subject to altered gait patterns which makes difficult to identify and to reliably detect gait events or compute gait parameters compared to healthy subjects. Different methods have been proposed based on wearable and non-wearable systems using multiple sensors [33]. Our aim has been to conceive a ready-to-use patient-oriented solution [34], using a minimum number of sensors and not requiring individual calibration or threshold parameterization for correctly triggering stimulation.

In Moore et al. work [5], the investigators monitored during $75 \mathrm{~min}$ the gait of subjects with Parkinson's Disease. For detecting locomotor activity, they have defined periods where the (Root Mean Square) RMS of the vertical acceleration was greater than $0.4 \mathrm{~m} / \mathrm{s}^{2}$ above baseline [35]. Hundza et al. [36] proposed a method for accurately and reliably detect gait cycle in PD. They used gyroscope angular rate reversal to identify the start of each gait cycle during walking. By interpolating zero-crossing of angular rate from a foot mounted IMU, they detected the termination of forward swing (TOFS) and consider it as physically close to heel strike. They defined it as the start and end point of the stride time for each gait cycle.

In our case, the strategy was to determine the feasibility of using one inertial sensor as a heel switch alternative in order to trigger stimulation. In future work, we will use information from the sensor to modulate the stimulation in real time, in case of FOG detection, u-turn or for example to adapt the stimulus to gait pattern. Such sensor may also be used to monitor some gait parameters in order to assess functional improvement [34], [37]-[39]. 
We aimed to detect stationary periods from the foot mounted IMU combining accelerometer and gyrometer measurements. In order to be the most sensitive and responsive to heel off velocity we chose to attach the IMU under the lateral malleolus. For defining lowest sensibility thresholds, we had to firstly filter raw inertial data. As the latency was a crucial parameter, we chose to use an Exponential Moving Average (EMA, low pass, Infinite Impulse Response - IIR) filter. At any time, the output of the filter $g X_{\text {filt }}$ is a weighted sum of the new sensor value $g X_{\text {raw }}$ and the old filter output $g X_{\text {filt }}$ old . Filter coefficient $\alpha$ controls the filtering effects:

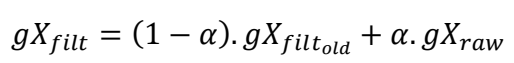

with $\alpha \in[0,1]$

Our data being processed at $100 \mathrm{~Hz}$, we designed a low pass filter of order 1 type butterworth with an attenuation of $3 \mathrm{~dB}$ at a cutoff frequency of $5 \mathrm{~Hz}$ using $\alpha=0.1367$ with only one sample late.

In motionless situation, the acceleration norm is supposed to be around $a c c_{t h}=9.81 \mathrm{~m} \cdot \mathrm{s}^{-2}$ (depending on sensor quality and calibration). From gyrometer angular profile, we determined a magnitude threshold, which is the limit between foot flat phase and heel off phase $\left(g y r_{t h} \cong 30 \mathrm{deg} / \mathrm{s}\right)$.

The difficulty was to be able to reliably detect stationary and non-stationary periods, in any kind of pathological gait without changing previously set thresholds. Combining accelerometer and gyrometer 3D norms of filtered data as defined in Equ. 2, we were able to successfully detect non-stationary periods on every PD subject. A maximum stimulation duration and a minimum successive stride time duration were also defined.

Based on Equ. 2, stimulation was triggered when a non-stationary period was detected.

$$
\text { if }\left\{\begin{array}{c}
\operatorname{norm}\left(\operatorname{acc}_{\text {filt }}\right) \leq a c c_{t h} \\
\text { and } \\
\text { norm }\left(\operatorname{gyr}_{\text {filt }}\right) \leq g y r_{t h}
\end{array}\right\} \Rightarrow>\text { stationary state }
$$

In order to validate our approach, we have analyzed offline the Start/End times of the stationary period as detected online through Equ. 2. As an example, in Fig. 2 we plotted the foot angular speed and the Start/Stop stimulation events triggered by the non-stationary period detection (NSPD) algorithm. Recorded from a real-time trial, it illustrates relatively to Heel-off and Swing phases that stimulation was actually initiated during heel off and terminated during swing phase. 


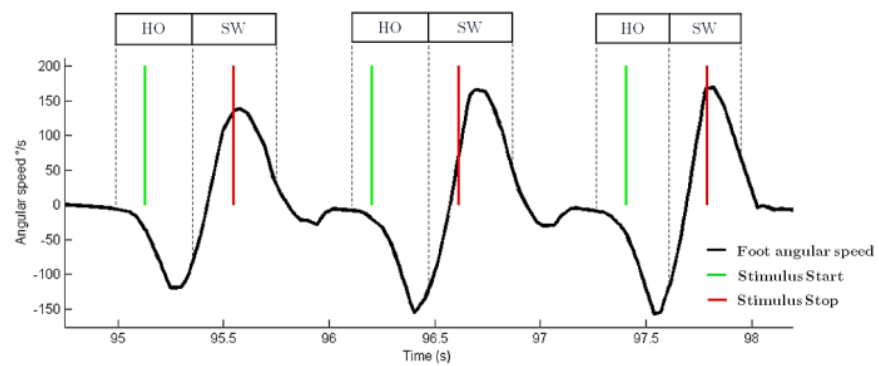

Figure 2 Example of real-time stimulation triggering based on Equ 2 from an experimental record. Green and red lines are respectively the start and stop stimulation events triggered by our non-stationary period detection algorithm. Heel off (HO) and swing (SW) phases were manually added to the figure for reference.

\section{Experimental Protocol}

13 subjects with Parkinson's disease (10 male, 3 female; Age range: 60 to 82 years) participated to the study. The protocol has been approved by local ethical committee (international identification number NCT02317289). Participants were recruited at the Neurology (Gui de Chauliac Hospital) and Gerontology (Balmes Center) departments of Montpellier hospital (CHU Montpellier). All subjects gave their informed written consent and were under the care of a neurologist also in charge of collecting clinical data.

Table 1 Clinical data of patients included in the study.

\begin{tabular}{|c|c|c|c|c|c|c|c|c|}
\hline ID & AGE & $\begin{array}{c}\text { DISEASE } \\
\text { DURATION }\end{array}$ & $\begin{array}{c}\text { STAGE } \\
(\mathrm{H} \& \mathrm{Y})\end{array}$ & $\begin{array}{c}\text { AGE } \\
\text { OF } \\
\text { ONSET }\end{array}$ & $\begin{array}{c}\text { *MDS- } \\
\text { UPDRS } \\
\\
3.11: \\
\text { FREEZING } \\
\text { / } 3.10: \\
\text { WALK / } \\
\text { GLOBAL } \\
\text { PART III }\end{array}$ & $\begin{array}{l}\text { Freezing } \\
\text { (Occasional } \\
\text { / Frequent) }\end{array}$ & $\begin{array}{l}\text { Falls } \\
(\mathrm{Y} / \mathrm{N})\end{array}$ & $* * \mathrm{MoCA}$ \\
\hline 1 & 71 & 5 & 2 & 66 & $1 / 1 / 28$ & $\mathrm{O}$ & $\mathrm{N}$ & 26 \\
\hline 2 & 63 & 7 & 3 & 56 & $1 / 1 / 28$ & $\mathrm{~F}$ & $\mathrm{Y}$ & 30 \\
\hline 3 & 71 & 18 & 3 & 53 & $2 / 2 / 40$ & $\mathrm{~F}$ & $\mathrm{Y}$ & 30 \\
\hline 4 & 74 & 22 & 3 & 52 & $1 / 2 / 23$ & $\mathrm{~F}$ & $\mathrm{Y}$ & 25 \\
\hline 5 & 72 & 7 & 3 & 65 & $2 / 2 / 28$ & $\mathrm{~F}$ & $\mathrm{Y}$ & 27 \\
\hline 6 & 74 & 8 & 3 & 48 & $3 / 3 / \mathrm{na}$ & $\mathrm{F}$ & $\mathrm{Y}$ & 12 \\
\hline 7 & 60 & 13 & 3 & 47 & $3 / 2 / 29$ & $\mathrm{~F}$ & $\mathrm{Y}$ & 25 \\
\hline 8 & 66 & 3 & 4 & 63 & $2 / 1 / 30$ & $\mathrm{~F}$ & $\mathrm{Y}$ & 23 \\
\hline 9 & 76 & 7 & 3 & 69 & $1 / 1 / 32$ & $\mathrm{~F}$ & $\mathrm{~N}$ & 23 \\
\hline 10 & 74 & 10 & 3 & 64 & $2 / 3 / 35$ & $\mathrm{~F}$ & $\mathrm{~N}$ & 21 \\
\hline 11 & 66 & 14 & 4 & 52 & $4 / 4 / 66$ & $\mathrm{~F}$ & $\mathrm{Y}$ & na \\
\hline 12 & 74 & 13 & 3 & 61 & $2 / 2 / 41$ & F & $\mathrm{Y}$ & 25 \\
\hline 13 & 82 & 15 & 3 & 67 & $1 / 3 / 47$ & $\mathrm{~F}$ & $\mathrm{Y}$ & 26 \\
\hline
\end{tabular}

*MDS-UPDRS: Movement Disorder Society-Unified Parkinson's Disease Rating Scale, from 0(normal) to 4(inability) **MoCA: MOntreal Cognitive Assessment, the maximum score is 30 points; a score of 26 or above is considered normal. 
The experimental protocol was divided into three parts. A) The first one consisted in asking the subjects to perform the SIP task. As described in Nantel et al. [40], the subjects had to stand $10 \mathrm{~s}$ on two force plates (Advance Mechanical Technology Inc, Watertown, MA, USA) with eyes open. The experimenter then gave a start signal and the subjects raised their legs alternately at a self-selected pace for $100 \mathrm{~s}$. All subjects were asked to perform the task twice. During the first trial, subjects who easily stepped in place without freezing were invited to repeat the SIP with a dual task (naming months of the year backwards while stepping). B) The second part of our protocol consisted in recording the time needed for the subject to stand up, to walk three meters, to do a u-turn, to come back and to sit down. This task is also known as "Timed Up and Go" (TUG) and described for the first time in Podsiadlo [41]. TUG task was repeated twice by the subjects. All trials were video-recorded for later manually labelling and timing each event. Parts A and B were designed for evaluating SIP and TUG as a possible tool for measuring FOG occurrence, gait performances and motor disorders. The aim was to better characterize subject's profile regarding responsivity to part C). The third and main part of the protocol consisted in assessing the use of electrical stimulation as a cueing method on the same population.
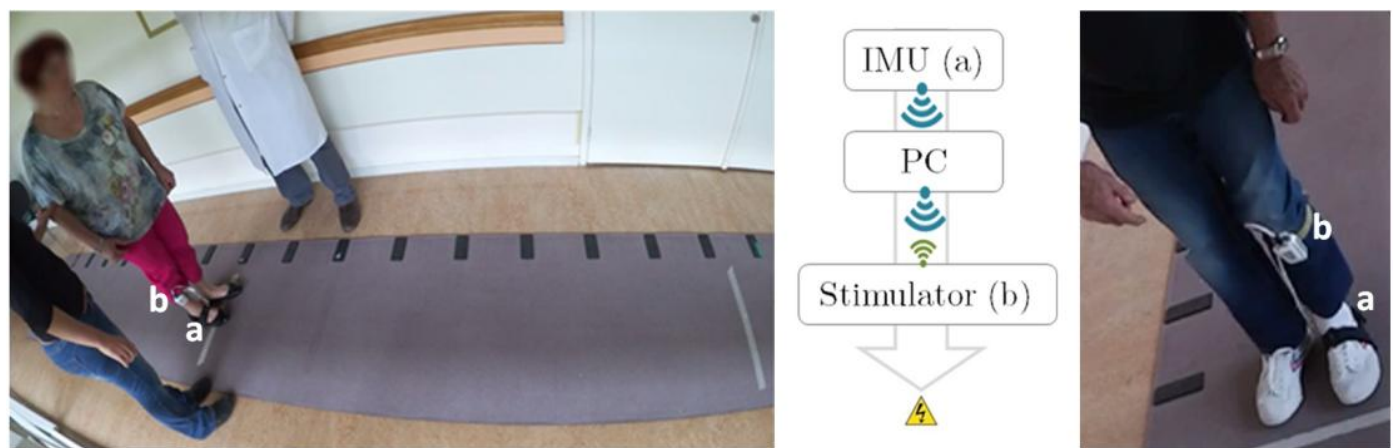

Figure 3 The subject is equipped with an inertial measurement unit (a) and a programmable stimulator (b) wirelessly connected through a PC.

Participants started from standing in the middle of a gait carpet. After a short familiarization to walk under stimulation, subjects were instructed to walk until reaching a line drawn on the ground, then do a u-turn, walk 5 meters, walk around a cone and keep walking to the start-stop line in the middle of the carpet. The test was repeated five times under the following conditions: no cueing pre-condition $(\mathrm{C} 0)$, stimulation cueing $(\mathrm{C} 1)$, no cueing postcondition (CObis). $\mathrm{C} 0$ is considered as the baseline. For eliminating learning bias, we asked the participants to perform a 10 min ecological path without stimulation (random walk in the hospital) between $\mathrm{C} 1$ and CObis conditions, to ensure in case of improvement in $\mathrm{C} 1$ that the performances went back to baseline level in CObis.

In each condition ( 5 trials per condition) of the stimulation protocol, we analyzed the last three trials. Each FOG event, u-turn execution times, 5-meters execution times and times to walk-around the cone were assessed from the video recording. 

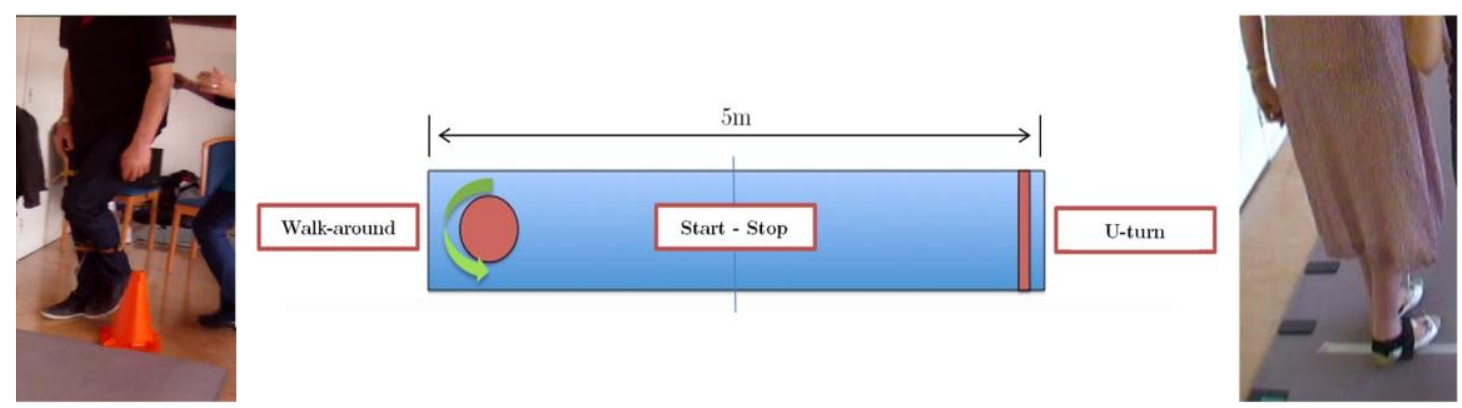

Figure 4 Five meters experimentation path. The subject starts in the middle of the walkway, walks 2,5 m, performs

a u-turn at the line, goes back $5 \mathrm{~m}$, walks around a cone, walks back 2,5 m then stops where he started.

\section{RESUlTS}

\section{A. Stepping in place}

For each participant, we analyzed from video recording the number of FOG events, their durations and the stepping cadence $10 \mathrm{~s}$ after the beginning and at the end of the SIP task (table 2). One patient was too exhausted to perform a second trial.

Table 2 Stepping In Place (SIP): Freezing Of Gait occurrence and cadence

\begin{tabular}{|c|c|c|c|c|c|c|c|c|c|c|}
\hline \multirow[b]{2}{*}{ ID } & \multicolumn{5}{|c|}{ SIP 1} & \multicolumn{5}{|c|}{ SIP 2} \\
\hline & $\begin{array}{l}\# \\
F \\
\text { O } \\
\text { G }\end{array}$ & $\begin{array}{c}\text { MEAN } \\
\text { FOG } \\
\text { DURATION } \\
\text { (s) }\end{array}$ & $\begin{array}{l}\text { DUAL } \\
\text { TASK } \\
(\mathrm{Y} / \mathrm{N})\end{array}$ & $\begin{array}{c}\text { CADENCE } \\
\mathrm{T}=10 \mathrm{~s} \\
\text { (step/min) }\end{array}$ & $\begin{array}{c}\text { CADENCE } \\
\mathrm{T}=110 \mathrm{~s} \\
\text { (step/min) }\end{array}$ & $\begin{array}{l}\# \\
\mathrm{~F} \\
\mathrm{O} \\
\mathrm{G}\end{array}$ & $\begin{array}{c}\text { MEAN } \\
\text { FOG } \\
\text { DURATION } \\
\text { (s) }\end{array}$ & $\begin{array}{l}\text { DUAL } \\
\text { TASK } \\
\text { (Y/N) }\end{array}$ & $\begin{array}{c}\text { CADENCE } \\
\mathrm{T}=10 \mathrm{~s} \\
(\mathrm{step} / \mathrm{min})\end{array}$ & $\begin{array}{c}\text { CADENCE } \\
\mathrm{T}=100 \mathrm{~s} \\
\text { (step/min) }\end{array}$ \\
\hline 1 & 0 & & $\mathrm{~N}$ & 65 & 70 & 0 & & $\mathrm{~N}$ & 70 & 75 \\
\hline 2 & 0 & & $\mathrm{~N}$ & 48 & 49 & 0 & & Y & 48 & 47 \\
\hline 3 & 0 & & $\mathrm{~N}$ & 36 & 60 & 0 & & $\mathrm{~N}$ & 42 & 60 \\
\hline 4 & 0 & & $\mathrm{~N}$ & 72 & 105 & 0 & & $\mathrm{~N}$ & 72 & 84 \\
\hline 5 & 0 & & $\mathrm{~N}$ & 72 & 70 & 3 & 2.0 & $\mathrm{Y}$ & 72 & 50 \\
\hline 6 & 0 & & $\mathrm{~N}$ & 96 & 110 & 0 & & $\mathrm{~N}$ & 96 & 96 \\
\hline 7 & 4 & 2.1 & $\mathrm{~N}$ & 60 & 70 & & & & & \\
\hline 8 & 8 & 2.3 & $\mathrm{~N}$ & 48 & 52 & 10 & 2.2 & $\mathrm{~N}$ & 50 & 50 \\
\hline 9 & 5 & 3.1 & $\mathrm{~N}$ & 58 & 60 & 0 & & $\mathrm{~N}$ & 48 & 50 \\
\hline 10 & 8 & 5.4 & $\mathrm{~N}$ & 110 & 105 & 9 & 3.1 & $\mathrm{~N}$ & 105 & 110 \\
\hline 11 & 8 & 3.2 & $\mathrm{~N}$ & 96 & 66 & 5 & 15.2 & $\mathrm{Y}$ & 100 & 96 \\
\hline 12 & 6 & 2.7 & $\mathrm{~N}$ & 108 & 120 & 10 & 6.1 & Y & 110 & 112 \\
\hline 13 & 6 & 3.3 & $\mathrm{~N}$ & 27 & 35 & 2 & 3.2 & $\mathrm{~N}$ & 36 & 36 \\
\hline
\end{tabular}

Only 7 of the 13 patients experienced FOG episodes during the first SIP task. One subject (ID5) who did not experience FOG during the first trial had FOG episodes in the second trial when performing a dual task. In all but one patient, the cadence at the end of the task was similar or above the cadence at the beginning. Some patients had difficulties to stay at the center of the platform during the task and were invited to change their position during the 
tasks. The height of step was highly variable among the different patients. The interruption of the SIP task observed in some patients was not due to freezing of gait but to fatigue, when participants were too exhausted to walk anymore. With dual task, the number of FOG events increased in 2 patients (ID5 and ID12) and decreased in one (ID11).

B. $T U G$

Table 3 Timed Up and Go (TUG): Freezing Of Gait occurrence and gait performances

\begin{tabular}{|c|c|c|c|c|c|c|c|c|c|c|}
\hline \multirow[b]{2}{*}{ ID } & \multicolumn{5}{|c|}{ TUG 1} & \multicolumn{5}{|c|}{ TUG 2} \\
\hline & $\begin{array}{l}\text { T.U.G. } \\
\text { DUR. } \\
\text { (s) }\end{array}$ & \# FOG & $\begin{array}{c}\text { FOG } \\
\text { MEAN } \\
\text { DURATION } \\
\text { (s) }\end{array}$ & $\begin{array}{l}\text { CADENCE } \\
\text { (step/min) }\end{array}$ & $\begin{array}{l}\text { AVG. } \\
\text { SPEED } \\
(\mathrm{km} / \mathrm{h})\end{array}$ & $\begin{array}{l}\text { T.U.G. } \\
\text { DUR. } \\
\text { (s) }\end{array}$ & \# FOG & $\begin{array}{c}\text { FOG } \\
\text { MEAN } \\
\text { DURATION } \\
(\mathrm{s})\end{array}$ & $\begin{array}{l}\text { CADENCE } \\
\text { (step/min) }\end{array}$ & $\begin{array}{l}\text { AVG. } \\
\text { SPEED } \\
(\mathrm{km} / \mathrm{h})\end{array}$ \\
\hline 1 & 10.1 & 0 & & 105 & 2.2 & 11.2 & 0 & & 110 & 2.0 \\
\hline 2 & 14.2 & 1 & 3.1 & 60 & 1.5 & 19.1 & 1 & 7.1 & 65 & 1.1 \\
\hline 3 & 16.7 & 1 & 2.3 & 60 & 1.4 & 13.1 & 1 & 2.2 & 90 & 1.7 \\
\hline 4 & 21.6 & 0 & & 65 & 1.0 & 19.2 & 0 & & 65 & 1.1 \\
\hline 5 & 15.5 & 0 & & 60 & 1.4 & 10.1 & 0 & & 60 & 2.2 \\
\hline 6 & 17.3 & 0 & & 65 & 1.3 & 14.2 & 0 & & 60 & 1.5 \\
\hline 7 & 13.1 & 2 & 2.6 & 65 & 1.7 & 12.6 & 2 & 2.5 & 60 & 1.8 \\
\hline 8 & 35.0 & 2 & 8.2 & 48 & 0.6 & 25.4 & 2 & 10.1 & 45 & 0.9 \\
\hline 9 & 22.2 & 1 & 3.4 & 50 & 1.0 & 21.8 & 1 & 3.2 & 48 & 1.0 \\
\hline 10 & 40.3 & 2 & 10.6 & 75 & 0.5 & 26.7 & 1 & 5.7 & 110 & 0.8 \\
\hline 11 & 30.6 & 2 & 1.2 & 80 & 0.7 & 22.2 & 3 & 3.4 & 100 & 1.0 \\
\hline 12 & 13.2 & 1 & 1.1 & 90 & 1.7 & 13.0 & 0 & & 96 & 1.7 \\
\hline 13 & 70.1 & 3 & 3.3 & 72 & 0.3 & & & & & \\
\hline
\end{tabular}

During the "Timed Up and Go" test, we assessed through video recording the total duration needed for the participants to accomplish the whole path, their cadence, average speed, and the FOG occurrences and durations (Table 3). Five participants over the 13 did not experience any FOG event during the TUG task. All the subjects without FOG during the TUG also did not have a FOG event during the SIP task. Twenty-six FOG events have been identified during the 2 trials of these 13 participants. 


\section{Stimulation}

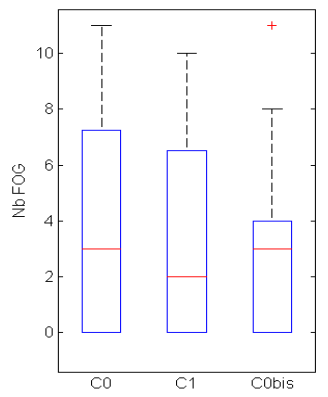

Figure 5 Number of Freezing of Gait events in baseline (C0), stimulation (C1) and control baseline (CObis) on all subjects $(n=13)$.

In a previous study [37], we observed that people with Parkinson's Disease scored with a high FOG-Q in daily life were not necessarily those who were prone to experience FOG during clinical experimental protocols. In table 1, we can see that subjects ID 2, 3 and 4 reported as frequent freezers in their daily life did not freeze in C0, neither during the SIP task and only once during the TUG task for the patient ID2. Thus we chose to classify the studied population in two groups, whether subjects experienced FOG in $\mathrm{C} 0$ or not.

In the "freezers group" $(\mathrm{n}=9)$, we observed that cueing globally decreased of $12 \%$ FOG occurrence compared to baseline without cueing. Table 4 shows cueing's effects in relation to baselines for all the participants and in each subgroup during the different experimental path phases. Cueing improved gait performances in all our subjects (Table 4). Considering all participants, we observed a reduction of $15 \%$ in turning time, $14 \%$ in 5 -m covering duration and $19 \%$ in time needed to walk-around the cone. In "freezers group", turning time is improved by $21 \%$, time to walk-around the cone is reduced by $25 \%$ and the duration needed to cover the $5-\mathrm{m}$ walk decreased by $18 \%$. The entire path is completed $19 \%$ shorter than baseline.

FOG repartition on all trials was four times more frequent during turning phases than when walking in a straight line, and two patients who did not freeze doing TUG and SIP task, actually froze in C0 (Fig. 6).

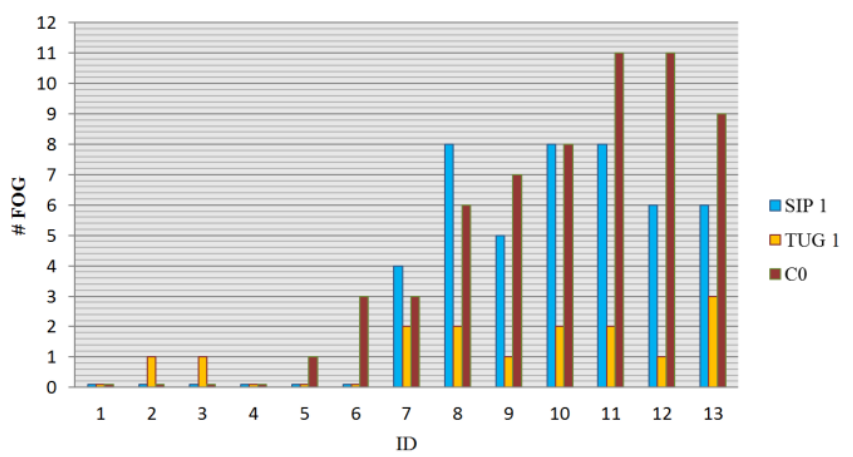

Figure 6 Number of FOG events between "Stepping In Place", "Timed Up and Go" and CO (stimulation protocol baseline). 
Table 4 Durations (standard deviation) of u-turn, 5-meters and Walk-around phases compared between baseline 1 (C0), stimulation (C1) and baseline 2 (CObis) in all the subjects $(n=13)$ and in the subgroups (freezers and non-freezers in $\mathrm{CO}$ ).

\begin{tabular}{|c|c|c|c|}
\hline & $\begin{array}{c}\text { All } \\
(\mathrm{N}=13)\end{array}$ & $\begin{array}{c}\text { Non Freezers } \\
(\mathrm{n}=4)\end{array}$ & $\begin{array}{c}\text { Freezers } \\
(\mathrm{n}=9)\end{array}$ \\
\hline \multicolumn{4}{|c|}{ U-Turn Time (s) } \\
\hline Baseline $1(\mathrm{C} 0)$ & $3.0(1.6)$ & $1.9(0.6)$ & $4.1(3.0)$ \\
\hline Stimulation (C1) & $2.6(1.1)$ & $1.8(0.6)$ & $3.4(2.7)$ \\
\hline Baseline 2 (CObis) & $3.2(1.3)$ & $2.2(0.9)$ & $4.1(3.0)$ \\
\hline \multicolumn{4}{|c|}{ Walk Around Time (s) } \\
\hline Baseline $1(\mathrm{C} 0)$ & $4.7(3.5)$ & $2.2(0.3)$ & $7.2(3.5)$ \\
\hline Stimulation (C1) & $3.8(2.3)$ & $2.1(0.4)$ & $5.4(4.1)$ \\
\hline Baseline 2 (CObis) & $4.7(3.5)$ & $2.2(0.3)$ & $7.2(6.5)$ \\
\hline \multicolumn{4}{|c|}{ 5m Time $(\mathrm{s})$} \\
\hline Baseline $1(\mathrm{C} 0)$ & $6.7(1.8)$ & $5.4(1.3)$ & $7.9(4.2)$ \\
\hline Stimulation (C1) & $5.8(1.3)$ & $4.9(1.6)$ & $6.7(2.1)$ \\
\hline Baseline 2 (CObis) & $6.6(1.6)$ & $5.4(0.8)$ & $7 . .7(3.3)$ \\
\hline
\end{tabular}

Two subjects (ID 7 and 13) showed a strong responsivity to the stimulation protocol. We decided to go further in analyzing their results (Fig. 7). Subject ID13 was one of the slowest participants as he needed more than $13 \mathrm{~s}$ to perform the $5 \mathrm{~m}$ path. This was reduced to $8 \mathrm{~s}$ with electrical stimulation based cueing in $\mathrm{C} 1$. Without stimulation he froze systematically doing the turning phase while he did not freeze at all in C1. Subject ID7 froze seven times during C0 protocol. With stimulation he froze only twice and the mean duration of his FOG events decreased from $5.5 \mathrm{~s}$ to $2.4 \mathrm{~s}$. However, the time to perform the $5 \mathrm{~m}$ path remained unchanged in both conditions. In previous works [34] we designed a stride length estimation algorithm validated on 12 participants with PD. From this preceding protocol we considered as significant a change in stride length greater than or equal to $20 \%$. Here we computed the stride length and cadence of subjects ID7 and ID13 from IMU data using the same algorithms. While there were no significant changes in stride length $(<10 \%$ increase) for both participants, the cadence of subject ID13 increased of $41 \%$ in $\mathrm{C} 1$ condition.
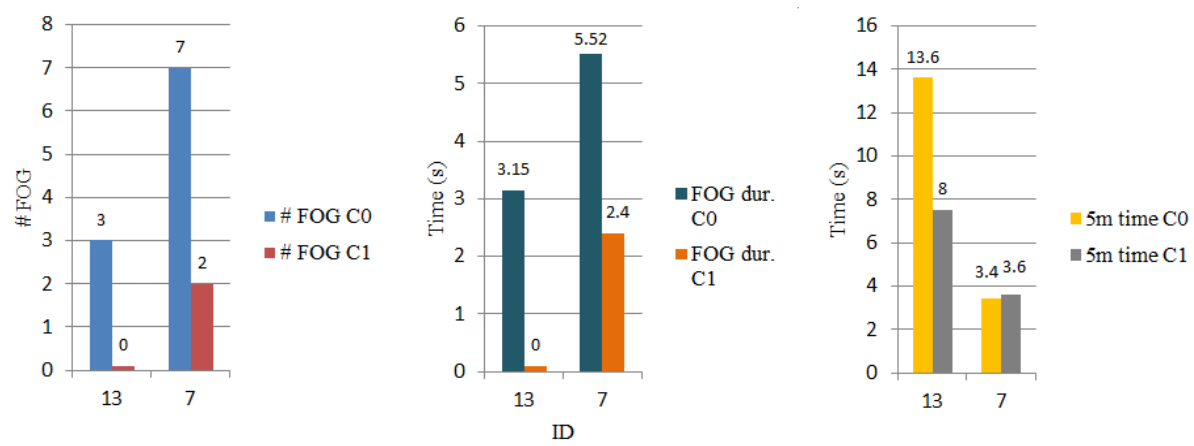

Figure 7 Subjects ID13 and ID7: FOG occurrence, FOG duration and time needed to walk 5 m. Comparison between C0 (baseline) and C1 (stimulation) 


\section{DISCUSSION}

Through this study, we investigated the feasibility of using electrical stimulation as a somatosensory cueing method of walking in Parkinson's disease. The aim was to investigate the capability of this cueing modality to prevent or at least reduce FOG events and to improve gait performances. As partly related to environmental triggers, assessing freezing of gait during a clinical protocol is challenging. In accordance with other studies, our comparative results show that there is some discrepancy between patient's self-assessment, SIP and walking task measurements. The occurrence of freezing episodes is highly variable in mild freezers and depends on the emotional context and on the tasks repetitions. It has been hypothesized that people with Parkinson's disease use attentional strategies to compensate for their gait impairment. This cognitive engagement could be insufficient in a cognitive dual task but could be helpful in a challenging condition, like a research experiment. In the latter case, the participant focuses on planning his walk trajectory. For instance, we noticed that some subjects could freeze repeatedly from the waiting room to the laboratory and rarely during the experiment. This discrepancy raises questions about the specificities that would need each cueing method. Thus, the effect of auditory rhythm on gait would seem to be directly correlated to stimuli properties. It would be superior when using non isosynchronous rhythm [42]. When louder, auditory stimuli could improve force [43]. Rythmic auditory cueing seems also to be effective on improving perceptual and motor timing [44] and can be used as a gait rehabilitation method in people with PD [45]. The meta-analysis done by Spaulding et al. [13] conclude that auditory cueing is more effective that visual cueing. The former improved stride length velocity while visual cueing only significantly improved stride length (Hedge g=.554; 95\% CI, .072-1.036). However there is no gold standard for evaluating the effect of interventions on FOG, which limits considerably comparative studies. From our results, the place of SIP as a FOG "biomarker" would need to be confirmed by other studies. In our case, the small number of FOG events identified led us to abandon this task for evaluating the effects of electrical stimulation. In addition, we consider the use of only force plates to be inadequate for detecting FOG events as described in [40]. The SIP task interruptions observed in some subjects and that could have been detected via the force data were often due to fatigue and not to FOG. For a better reliability, this observation led us to label FOG events from video, instead of using only force data.

Based on previous observations and literature, we designed a protocol with turning phases in order to increase FOG events occurrence. This hypothesis has been validated as FOG repartition on all trials was four times more frequent during turning phases than when walking in a straight line and two patients who did not freeze doing TUG and SIP task, actually froze in C0 (Fig. 6).

Our results show a global positive effect on gait performances, as the time needed to achieve the protocol was considerably shorter with stimulation cueing. "Freezer" patients tend to be more responsive to cueing, with a turning time improved by $21 \%$. We also observe a $12 \%$ decrease in FOG occurrence compared to baseline. However, the size 
of the studied population was too small for showing a statistically significant effect. Extra experiments will be done to include more participants.

None of the participants reported uncomfortable sensations induced by electrical stimulation. Some of them expressed an interest in such a possibility to be helped while walking in their daily-life and seemed to accept the additional technological equipment arising from it. In common with auditory and visual stimuli in other studies, sensitivity to the electrical cueing differs among the patients. We noticed that the electrical stimulation threshold needed for feeling the stimulus was highly variable between patients. The absence of quantitative assessment of the electrical stimulation effect was problematic as we needed to rely on patient feedback while knowing their sensory perception may be altered. Two of our 13 participants have been clearly improved by the stimulation (respectively $85 \%$ of FOG events reduction and a 5-m path $42 \%$ shorter compared to baseline for one), while it did not affect at all some others. In any cases, stimulation cueing never worsen performances or FOG occurrence.

In this protocol, the use of an inertial sensor based trigger did not offer much more functionality than a basic heel switch. However, having access to gait kinematics data [34], [39] and to path information from only one sensor could be useful to real-time adapt cueing, when for example a turning phase or a FOG event [26], [37] is detected by the sensor. We could also modulate stimulation or dynamically change the trigger timing regarding walking rhythm or external events. Many other triggering strategies could be investigated and some technical aspects need to be improved for getting rid of some latency problems we experienced during the trials. Another approach could also consist in eliciting different responses by testing various electrode locations under the foot [46].

Due to technical constraints, we could only equip one leg of our subject. We asked them which direction of rotation was the most difficult for them and we equipped the most problematic limb. Considering the stimulation side may affect the observed effects in asymmetric tasks, part of our upcoming experiments will also involve bilateral stimulation.

\section{CONCLUSION}

This preliminary study suggests that a somatosensory cueing modality based on sensitive electrical stimulation could be a promising option for improving gait of patients with Parkinson Disease. Experimental results brought to light a favorable benefit on both spatiotemporal gait parameters and FOG occurrence. Further work is needed to improve the modalities of cueing strategy before initiating a larger comparative study with other cueing methods usually developed in PD. As observed with SIP protocol, a global methodology for effectively and reliably assessing cueing effects and FOG in a clinical environment remains still to be investigated.

\section{ACKNOWLEDGMENT}

This work was supported by an INRIA internal financial support: ADT SENSBIO and a Montpellier Hospital internal financial support (AOI 9096 DEMAR-PARK CHU Montpellier). 
[1] J. Vandenbossche, N. Deroost, E. Soetens, D. Coomans, J. Spildooren, S. Vercruysse, a Nieuwboer, and E. Kerckhofs, "Freezing of gait in Parkinson's disease: disturbances in automaticity and control.," Front. Hum. Neurosci., vol. 6, no. January, p. 356, 2012.

[2] J. Jankovic, "Parkinson's disease : clinical features and diagnosis," Neurol Neurosurg Psychiatry, vol. 79, no. 1957 , pp. 368-376, 2008.

[3] B. R. Bloem, J. M. Hausdorff, J. E. Visser, and N. Giladi, "Falls and freezing of Gait in Parkinson's disease: A review of two interconnected, episodic phenomena," Mov. Disord., vol. 19, no. 8, pp. 871-884, 2004.

[4] E. Heremans, A. Nieuwboer, and S. Vercruysse, "Freezing of gait in Parkinson's disease: where are we now?," Curr. Neurol. Neurosci. Rep., vol. 13, no. 6, p. 350, 2013.

[5] S. T. Moore, H. G. MacDougall, J. M. Gracies, H. S. Cohen, and W. G. Ondo, "Long-term monitoring of gait in Parkinson's disease," Gait Posture, vol. 26, no. 2, pp. 200-207, 2007.

[6] S. T. Moore, H. G. MacDougall, and W. G. Ondo, "Ambulatory monitoring of freezing of gait in Parkinson's disease,” J. Neurosci. Methods, vol. 167, no. 2, pp. 340-348, Jan. 2008.

[7] J. M. Rahman S, Griffin HJ, Quinn NP, "The factors that induce or overcome freezing of gait in Parkinson's disease.," Neurol., vol. 19(3):127-, 2008.

[8] T. C. Rubinstein, N. Giladi, and J. M. Hausdorff, "The power of cueing to circumvent dopamine deficits: A review of physical therapy treatment of gait disturbances in Parkinson's disease," Mov. Disord., vol. 17, no. 6, pp. 1148-1160, 2002.

[9] E. Jovanov, E. Wang, L. Verhagen, M. Fredrickson, and R. Fratangelo, "deFOG - A real time system for detection and unfreezing of gait of Parkinson's patients," Proc. 31st Annu. Int. Conf. IEEE Eng. Med. Biol. Soc. Eng. Futur. Biomed. EMBC 2009, pp. 5151-5154, 2009.

[10] Y. Baram, "Virtual Sensory Feedback for Gait Improvement in Neurological Patients.," Front. Neurol., vol. 4, no. October, p. 138, 2013.

[11] M. Suteerawattananon, G. S. Morris, B. R. Etnyre, J. Jankovic, and E. J. Protas, "Effects of visual and auditory cues on gait in individuals with Parkinson's disease," J. Neurol. Sci., vol. 219, no. 1-2, pp. 63-69, 2004.

[12] M. H. Thaut, G. C. McIntosh, R. R. Rice, R. a Miller, J. Rathbun, and J. M. Brault, "Rhythmic auditory stimulation in gait training for Parkinson's disease patients.," Mov. Disord., vol. 11, no. 2, pp. 193-200, 1996.

[13] S. J. Spaulding, B. Barber, M. Colby, B. Cormack, T. Mick, and M. E. Jenkins, "Cueing and gait improvement among people with Parkinson's disease: A meta-analysis," Arch. Phys. Med. Rehabil., vol. 94, no. 3, pp. 562-570, 2013.

[14] E. van Wegen, C. de Goede, I. Lim, M. Rietberg, A. Nieuwboer, A. Willems, D. Jones, L. Rochester, V. Hetherington, H. Berendse, J. Zijlmans, E. Wolters, and G. Kwakkel, "The effect of rhythmic somatosensory cueing on gait in patients with Parkinson's disease," J. Neurol. Sci., vol. 248, no. 1-2, pp. 210-214, 2006.

[15] L.-A. Leow, T. Parrott, and J. A. Grahn, "Individual differences in beat perception affect gait responses to low- and high-groove music.," Front. Hum. Neurosci., vol. 8, no. October, p. 811, 2014.

[16] M. Vaugoyeau, S. Viel, C. Assaiante, B. Amblard, and J. P. Azulay, "Impaired vertical postural control and proprioceptive integration deficits in Parkinson's disease,” Neuroscience, vol. 146, no. 2, pp. 852-863, 2007.

[17] S. Mesure, J. P. Azulay, J. Pouget, and B. Amblard, "Strategies of segmental stabilization during gait in Parkinson's disease,” Exp. Brain Res., vol. 129, no. 4, pp. 573-581, 1999.

[18] F. Qiu, M. H. Cole, K. W. Davids, E. M. Hennig, P. A. Silburn, H. Netscher, and G. K. Kerr, "Effects of textured insoles on balance in people with Parkinson's disease," PLoS One, vol. 8, no. 12, 2013.

[19] G. E. Mann, S. M. Finn, and P. N. Taylor, "A pilot study to investigate the feasibility of electrical stimulation to assist gait in Parkinson's disease.," Neuromodulation, vol. 11, no. 2, pp. 143-9, 2008.

[20] L. Popa and P. Taylor, "Functional electrical stimulation may reduce bradykinesia in Parkinson' s disease : A feasibility study," vol. 0, no. 0, pp. 1-8, 2015.

[21] M. Djurić-Jovičić, S. Radovanović, I. Petrović, C. Azevedo, G. Mann, and M. B. Popović, "The impact of functional electrical stimulation (FES) on freezing of gait (FOG) in patients with Parkinson's disease," Clin. Neurophysiol., vol. 124, no. 7, p. e11, Jul. 2013.

[22] E. Khudados, F. W. J. Cody, and D. J. O'Boyle, "Proprioceptive regulation of voluntary ankle movements, demonstrated using muscle vibration, is impaired by Parkinson's disease," J. Neurol. Neurosurg. Psychiatry, vol. 67, no. 4, pp. 504-510, 1999.

[23] M. S. El-Tamawy, M. H. Darwish, and M. E. Khallaf, "Effects of augmented proprioceptive cues on the parameters of gait of individuals with Parkinson's disease.," Ann. Indian Acad. Neurol., vol. 15, no. 4, pp. 267-72, 2012.

[24] A. Kleiner, M. Galli, M. Gaglione, D. Hildebrand, P. Sale, G. Albertini, F. Stocchi, and M. F. De Pandis, "The Parkinsonian Gait Spatiotemporal Parameters Quantified by a Single Inertial Sensor before and after Automated Mechanical Peripheral Stimulation Treatment," Parkinsons. Dis., vol. 2015, 2015. 
[25] J. D. Schaafsma, Y. Balash, T. Gurevich, A. L. Bartels, J. M. Hausdorff, and N. Giladi, "Characterization of freezing of gait subtypes and the response of each to levodopa in Parkinson's disease," Eur. J. Neurol., vol. 10, no. 4, pp. 391-398, 2003.

[26] C. A. Coste, B. Sijobert, R. Pissard-Gibollet, M. Pasquier, B. Espiau, and C. Geny, "Detection of freezing of gait in Parkinson disease: preliminary results.," Sensors (Basel)., vol. 14, no. 4, pp. 6819-27, Jan. 2014.

[27] M. Plotnik, N. Giladi, Y. Balash, C. Peretz, and J. M. Hausdorff, "Is freezing of gait in Parkinson's disease related to asymmetric motor function?," Ann. Neurol., vol. 57, no. 5, pp. 656-663, 2005.

[28] P. Crenna, I. Carpinella, M. Rabuffetti, E. Calabrese, P. Mazzoleni, R. Nemni, and M. Ferrarin, “The association between impaired turning and normal straight walking in Parkinson's disease," Gait Posture, vol. 26, no. 2, pp. 172-178, 2007.

[29] A. Nieuwboer, K. Baker, A.-M. Willems, D. Jones, J. Spildooren, I. Lim, G. Kwakkel, E. Van Wegen, and L. Rochester, "The short-term effects of different cueing modalities on turn speed in people with Parkinson's disease.," Neurorehabil. Neural Repair, vol. 23, no. 8, pp. 831-836, 2009.

[30] J. Nantel, C. de Solages, and H. Bronte-Stewart, "Repetitive stepping in place identifies and measures freezing episodes in subjects with Parkinson's disease," Gait Posture, vol. 34, no. 3, pp. 329-333, 2011.

[31] R. J. Reed-Jones, M. A. Hollands, J. G. Reed-Jones, and L. A. Vallis, "Visually evoked whole-body turning responses during stepping in place in a virtual environment," Gait Posture, vol. 30, no. 3, pp. 317-321, 2009.

[32] E. G. Spaich, N. Svaneborg, H. R. Jørgensen, and O. K. Andersen, "Rehabilitation of the hemiparetic gait by nociceptive withdrawal reflex-based functional electrical therapy: a randomized, single-blinded study.," $J$. Neuroeng. Rehabil., vol. 11, p. 81, 2014.

[33] A. Muro-de-la-Herran, B. García-Zapirain, and A. Méndez-Zorrilla, "Gait analysis methods: An overview of wearable and non-wearable systems, highlighting clinical applications," Sensors (Switzerland), vol. 14, no. 2. pp. 3362-3394, 2014.

[34] B. Sijobert, M. Benoussaad, J. Denys, R. Pissard-gibollet, and C. A. Coste, "Implementation and validation of a stride length estimation algorithm, using a single inertial sensor on healthy and Parkinson' s disease subjects," Health (Irvine. Calif)., vol. 13, no. 9, pp. 1-7, 2015.

[35] H. G. MacDougall and S. T. Moore, "Marching to the beat of the same drummer: the spontaneous tempo of human locomotion.," J. Appl. Physiol., vol. 99, no. 3, pp. 1164-1173, 2005.

[36] S. R. Hundza, W. R. Hook, C. R. Harris, S. V. Mahajan, P. A. Leslie, C. A. Spani, L. G. Spalteholz, B. J. Birch, D. T. Commandeur, and N. J. Livingston, “Accurate and reliable gait cycle detection in parkinson's disease," IEEE Trans. Neural Syst. Rehabil. Eng., vol. 22, no. 1, pp. 127-137, 2014.

[37] B. Sijobert, J. Denys, C. A. Coste, and C. Geny, "IMU based detection of freezing of gait and festination in Parkinson's disease," in 2014 IEEE 19th International Functional Electrical Stimulation Society Annual Conference, IFESS 2014 - Conference Proceedings, 2014.

[38] C. Azevedo Coste, B. Sijobert, J. Froger, and C. Fattal, "Preliminary developments towards closed-loop FES-assistance of posture and gait," IFAC Proc. Vol., vol. 48, no. 20, pp. 333-337, 2015.

[39] M. Benoussaad, B. Sijobert, K. Mombaur, and C. Azevedo-Coste, "Robust Foot Clearance Estimation based on Acceleration Integration of Mounted Inertial Measurement Unit," Sensors, no. October 2015, pp. 1-13, 2015.

[40] J. Nantel, C. de Solages, and H. Bronte-Stewart, "Repetitive stepping in place identifies and measures freezing episodes in subjects with Parkinson's disease," Gait Posture, vol. 34, no. 3, pp. 329-333, Jul. 2011.

[41] D. Podsiadlo and S. Richardson, "The timed 'Up \& Go': a test of basic functional mobility for frail elderly persons," J. Am. Geriatr. Soc., vol. 39, no. 2, pp. 142-148, 1991.

[42] D. G. Dotov, S. Bayard, V. Cochen de Cock, C. Geny, V. Driss, G. Garrigue, B. Bardy, and S. Dalla Bella, "Biologically-variable rhythmic auditory cues are superior to isochronous cues in fostering natural gait variability in Parkinson's disease," Gait Posture, vol. 51, pp. 64-69, 2017.

[43] A. Anzak, H. Tan, A. Pogosyan, A. Djamshidian, H. Ling, A. Lees, and P. Brown, "Improvements in rate of development and magnitude of force with intense auditory stimuli in patients with Parkinson's disease," Eur. J. Neurosci., vol. 34, no. 1, pp. 124-132, 2011.

[44] C.-E. Benoit, S. Dalla Bella, N. Farrugia, H. Obrig, S. Mainka, and S. a. Kotz, "Musically Cued GaitTraining Improves Both Perceptual and Motor Timing in Parkinsonâ $€^{\mathrm{TM}_{\mathrm{s}}}$ Disease," Front. Hum. Neurosci., vol. 8, no. July, p. 494, 2014.

[45] A. Ashoori, D. M. Eagleman, and J. Jankovic, "Effects of auditory rhythm and music on gait disturbances in Parkinson's disease," Frontiers in Neurology, vol. 6, no. NOV. 2015.

[46] E. G. Spaich, L. Arendt-Nielsen, and O. K. Andersen, "Modulation of lower limb withdrawal reflexes during gait: a topographical study.," J. Neurophysiol., vol. 91, no. 1, pp. 258-66, 2004. 